\title{
REMUNERAÇÃO VARIÁVEL, UM ESTUDO DA RELAÇÃO COM INDICADORES FINANCEIROS DAS COMPANHIAS ABERTAS BRASILEIRAS*
}

\author{
JONATAN MARLON KONRAHT***, ROGÉRIO JOÃO LUNKES ${ }^{* * * *}$, VALDIRENE GASPARETTO***** \\ \& DARCI SCHNORRENBERGER $*$ **:*** \\ UNIVERSIDADE FEDERAL DE SANTA CATARINA (UFSC) (BRASIL)
}

Recibido/ Received/ Recebido: 08/03/2016 - Aceptado/ Accepted / Aprovado: 06/10/2016

\begin{abstract}
Resumo
A remuneração variável aos diretores é utilizada com o fim de alinhar os interesses destes e aqueles dos proprietários, contribuindo na melhora do desempenho organizacional. Neste contexto, este estudo tem como objetivo identificar se a percentagem de remuneração variável concedido aos diretores tem relação com o desempenho organizacional. Para tal fim, foram analisadas 75 empresas não financeiras que integram o Índice Brasil ( $\mathrm{IBrX}$ - 100) no período compreendido entre 2010 e 2014 para um total de 352 observações. Os dados foram coletados do formulário de referência e analisados usando o software Economatica ${ }^{\circledR}$ mediante uma regressão com dados de painel. Os resultados indicam que não existe relação estatisticamente significativa entre a percentagem oferecida de remuneração variável e o desempenho organizacional. A implicação prática deste resultado, é que para a mostra analisada, a atribuição de uma maior percentagem de remuneração variável não gera um impacto direto sobre o desempenho econômico e financiero das organizações.
\end{abstract}

Palabras clave: Remuneração; desempenho; indicadores financeiros; Brasil.

* Este artigo é baseado em uma pesquisa científica realizada pelos autores.

* Mestre em Contabilidade pela Universidade Federal de Santa Catarina (UFSC). Bacharel em Ciências Contábeis pela Universidade Estadual do Oeste do Paraná (UNIOESTE).

*** Pós-Doutorado pela Universidade de Valência (ESP). Doutor e Mestre em Engenharia de Produção pela UFSC. Bacharel em Ciências Contábeis pela UFSC. Professor do Programa de Pós-graduação em Contabilidade da Universidade Federal de Santa Catarina (UFSC).

Doutora e Mestre em Engenharia de Produção pela UFSC. Bacharel em Ciências Contábeis pela Universidade do Contestado (UNC). Professora do Programa de Pós-graduação em Contabilidade da Universidade Federal de Santa Catarina (UFSC).

Doutor e Mestre em Engenharia de Produção pela UFSC. Bacharel em Ciências Contábeis pela UFSC. Professor do Programa de Pós-graduação em Contabilidade da Universidade Federal de Santa Catarina (UFSC). 


\title{
RETRIBUCIÓN VARIABLE, UN ESTUDIO DE SU RELACIÓN CON LOS INDICADORES FINANCIEROS DE LAS COMPAÑÍAS BRASILEÑAS COTIZADAS EN BOLSA
}

\begin{abstract}
Resumen
La retribución variable a los directivos es utilizada con el fin de alinear los intereses de los directivos y propietarios, contribuyendo a mejorar el desempeño organizacional. En este contexto, este estudio tiene como objetivo identificar si el porcentaje de remuneración variable concedido a los directivos tiene relación con el desempeño organizacional. Para tal fin, fueron analizadas 75 empresas no financieras que integran el Îndice Brasil ( $\mathrm{IBrX}$ - 100) en el periodo comprendido entre 2010 y 2014 para un total de 352 observaciones. Los datos fueron recolectados del formulario de referencia y se analizaron usando el software Economatica ${ }^{\circledR}$ mediante una regresión con datos de panel. Los resultados indican que no existe relación estadísticamente significativa entre el porcentaje ofrecido de remuneración variable y el desempeño organizacional. La implicación práctica de este hallazgo, es que para la muestra analizada, la asignación de un mayor porcentaje de remuneración variable no genera un impacto directo sobre el desempeño económico y financiero de las organizaciones.
\end{abstract}

Keywords: Remuneración; Desempeño; Indicadores financieros; Brasil.

\section{VARIABLE REMUNERATION, A STUDY OF ITS RELATIONSHIP WITH THE BRAZILIAN COMPANIES FINANCIAL INDICATORS LISTED ON THE STOCK MARKET}

\begin{abstract}
The variable remuneration to managers is used in order to align the interests of managers and owners, contributing to improve organizational performance. In this context, this study aims to identify if the percentage of variable remuneration granted to managers is related to organizational performance. For this purpose, 75 non-financial companies that integrate the Brazil Index (IBrX - 100) in the period between 2010 and 2014 were analyzed for a total of 352 observations. The data were collected from the reference form and analyzed using the Economatica ${ }^{\circledR}$ software by regression with panel data. The results indicate that there is no statistically significant relationship between the percentage offered of variable remuneration and organizational performance. The practical implication of this finding is that for the analyzed sample, the assignment of a higher percentage of variable remuneration does not generate a direct impact on the economic and financial performance of the organizations.
\end{abstract}

Palavras chave: Remuneration; Performance; Financial indicators; Brazil.

Konraht, J., Lunkes, R., Gasparetto, V. \& Schnorrenberger, D. (2018) Remuneração variável: um estudo da relação com indicadores financeiros das companhias abertas brasileiras. En: Revista de la Facultad de Ciencias Económica: Investigación y Reflexión. rev.fac.cienc.econ, XXVI (1), DOI: https://doi.org/10.18359/rfce.1781

JEL: J30, M52. 


\section{Introdução}

Na perspectiva da Teoria da Agência, decorrente da separação entre propriedade e controle, a performance da companhia pode ser comprometida pelo desalinhamento de interesses entre proprietários e gestores (Jensen \& Meckling, 1976). Uma das formas de alinhar estes interesses é por meio da vinculação da remuneração do gestor ao alcance de metas preestabelecidas pelos proprietários (Smith \& Watts, 1982; Eisenhardt, 1989; Jensen \& Murphy, 1990a; Hirshleifer \& Suh, 1992).

Este tipo de remuneração variável (seu montante varia em função do desempenho) tende a afetar o comportamento dos gestores, pois influi sobre a sua aversão ao risco e comprometimento com a organização. Assim, incentiva-os a buscar maximizar o resultado das suas atividades e, consequentemente, o desempenho da empresa (Smith \& Watts, 1982; Hirshleifer \& Suh, 1992).

Esta situação é explicada pelo fato de que, para o gestor maximizar a sua riqueza (remuneração), precisa atingir as metas fixadas pela organização. Consequentemente, a riqueza do gestor está atrelada à riqueza dos proprietários. Esta situação cria incentivos ao gestor para a obtenção de desempenho superior nas suas atividades e, por conseguinte, melhorar o desempenho da organização em que atua.

Diante disto, pode-se assumir a hipótese de que este efeito-incentivo pode ser tanto maior quanto for o percentual da remuneração atrelada ao desempenho da empresa. Isto ocorre, pois, quanto maior é o percentual de remuneração baseada no desempenho, mais dependente a remuneração do gestor é do alcance das metas estabelecidas pela organização.

Porém, a validade desta hipótese no contexto brasileiro ainda carece de evidências empíricas. Assim, para verificar se esta hipótese de fato se faz presente nas organizações, formula-se a seguinte questão de pesquisa: existe relação entre o percentual de remuneração variável dos executivos e o desempenho econômico e financeiro das organizações?
O objetivo deste estudo é identificar se o percentual de remuneração variável oferecido ao gestor está relacionado com o desempenho econômico e financeiro das empresas. A premissa adotada nesta pesquisa é de que a forma como a remuneração é estrutura gera incentivos aos gestores maximizarem o desempenho da empresa. Assim, à medida que se eleva o percentual de remuneração baseada no desempenho (variável), tenderia a aumentar o incentivo dos gestores para o alcance das metas da empresa.

Cabe ressaltar que uma corrente da literatura defende que o montante da remuneração variável também pode influenciar o comportamento do gestor. No entanto, neste estudo optou-se pela análise do percentual de remuneração (estrutura da remuneração), pois a utilização do montante poderia conter problemas de endogeneidade com os indicadores analisados, uma vez que o montante da remuneração é resultado de uma equação que toma por base o desempenho.

Este estudo se justifica, pois constata-se, nas pesquisas realizadas no contexto brasileiro, o predomínio da análise da relação entre o montante de remuneração total dos executivos e o desempenho das organizações. Assim, este estudo oferece contribuições teóricas, pois analisa a relação entre o sistema de remuneração e o desempenho sob um ângulo ainda não explorado pela literatura nacional, o que amplia o entendimento sobre esta temática e contribui para a solidificação dos resultados encontrados na literatura nacional.

No tocante à literatura internacional, estudos anteriores realizados nos Estados Unidos indicam que, dentre os fatores da política de remuneração que possuem relação com o desempenho organizacional, a forma (fixa/variável) como a remuneração é composta exerce influência maior do que o total de remuneração paga (Jensen \& Murphy, 1990a; Mehran, 1995). Diante disto, esta pesquisa preenche uma lacuna teórica sobre a validade destas evidências para o contexto institucional brasileiro, o que contribui, na prática organizacional, para a elaboração de pacotes de remuneração que gerem incentivos para os profissionais ampliarem o desempenho das organizações. 


\section{Referencial teórico}

\subsection{Conflitos de interesses, estrutura de remune- ração e desempenho organizacional}

Um relacionamento de agência ocorre quando o proprietário (principal) delega o poder de decisão sobre seu patrimônio a um terceiro (agente), com a finalidade de que este atue na maximização da riqueza daquele. Esta situação implica na separação entre propriedade e controle (Jensen \& Meckling, 1976).

Como consequência, tem-se a origem dos problemas gerais de agência, uma vez que agente e principal tendem naturalmente a possuir interesses divergentes sobre a maximização da sua riqueza particular. Assim, o gestor, ao buscar a maximização da sua utilidade, pode tomar decisões que não atendam aos desejos do principal (contratante), embora tenha sido contratado para tal finalidade (Jensen \& Meckling, 1976).

Na perspectiva empresarial, um dos fatores que explicam este desalinhamento de interesses entre administradores e proprietários é o comportamento de maior aversão ao risco do agente em relação ao principal (Eisenhardt, 1989; Mehran, 1995). Este comportamento existe, pois os agentes, na posição de contratados, são incapazes de diversificar os seus riscos do contrato (emprego, prestígio etc.), enquanto os proprietários podem possuir investimentos diversificados e, assim, gerenciar o seu nivel de risco (Smith \& Stulz, 1985; Eisenhardt, 1989).

A aversão ao risco, no entanto, pode ser influenciada pela estrutura de remuneração da firma (Smith \& Watts, 1982; Hirshleifer \& Suh, 1992). Ademais, estes incentivos financeiros (remuneração) tendem a influenciar o nível de esforço despedido pelos gestores, o qual tem reflexo sobre o desempenho organizacional da firma (Bonner \& Sprinkle, 2002).

A estrutura de remuneração executiva comumente é composta por quatro elementos básicos: salário-base, bônus anual vinculado à performance contábil, stock options e planos de incentivos de longo prazo (Murphy, 1999)executive stock options, and option valuation.

Estes componentes podem ser categorizados em duas classes, em relação ao desempenho dos gestores: fixa e variável. O salário-base é um componente fixo da estrutura de remuneração, assim, não está vinculado ao desempenho alcançado. Os outros três componentes são classificados como variáveis, pois seu valor está associado à obtenção de certo nível de desempenho nas atividades do gestor (Murphy, 1999; Aguiar et al., 2012).

Quando a remuneração dos gestores é baseada apenas em salário fixo, estes tendem a tomar decisões de investimento mais conservadoras (Smith \& Watts, 1982). Especificamente, os agentes tendem a priorizar projetos de investimento que apresentem menor incerteza quanto aos fluxos de caixa futuros do que projetos que apresentem maiores valores presentes líquidos $e$, consequentemente, sejam mais arriscados (Smith \& Watts, 1982).

Assim, por conta de o gestor priorizar os projetos pela sua maior segurança e não pelo seu potencial de geração de valor (valor presente líquido), a maximização do desempenho da organização pode não ser alcançada.

Neste sentido, a literatura apresenta que uma das maneiras de se mitigar este problema decorrente da aversão ao risco é relacionar a remuneração do gestor ao desempenho da organização, a qual pode ser realizada por meio da remuneração variável (Smith \& Watts, 1982; Eisenhardt, 1989; Jensen \& Murphy, 1990a; Hirshleifer \& Suh, 1992). A remuneração baseada no desempenho tende a incentivar os gestores a investirem em projetos que maximizem a performance da firma, pois, consequentemente, isto aumenta a sua riqueza pessoal (Smith \& Watts, 1982).

Esta proposição teórica é corroborada empiricamente por Jensen \& Murphy (1990a) e Mehran (1995) que, ao analisarem as companhias norte-americanas, constataram que a forma como a remuneração é estruturada, ao invés do total da remuneração paga, afeta a motivação dos gestores para a maximização do desempenho das empresas. 
Ademais, o estabelecimento de contratos de remuneração baseados no desempenho do gestor tem o objetivo de servir como um mecanismo de alinhamento de interesses entre gestores e proprietários (Ittner, Larcker \& Rajan, 1997; Reichelstein, 2000; Beuren, Silva \& Mazzioni, 2014). Estes sistemas de incentivos tendem a reduzir os custos de agência, pois induzem os gestores a atuarem de acordo com as metas organizacionais estabelecidas, o que tende a maximizar o desempenho da organização (Merchant, Van Der Stede \& Zheng, 2003).

Uma das formas de remuneração variável que tem recebido extensivo interesse da literatura é a remuneração baseada em ações. Jensen \& Murphy (1990a) consideram que tornar o gestor proprietário de uma parcela da empresa é a maneira mais eficiente de se conectar os objetivos econômicos de gestores e proprietários. Hirshleifer \& Suh (1992) complementam afirmando que a remuneração baseada em ações (stock options) tem o efeito de reduzir os custos de agência decorrentes da aversão ao risco dos gestores, bem como os decorrentes do menor esforço despendido para a realização das atividades.

\subsection{Estudos anteriores}

Em nível internacional, os estudos empíricos sobre a relação entre remuneração dos gestores e desempenho organizacional são amplamente documentados. Inicialmente, estes estudos buscaram identificar a correlação entre o montante da remuneração dos executivos e o desempenho organizacional, bem como a sensitividade da remuneração, isto é, qual a variação do montante recebido pelos executivos em função da variação do desempenho organizacional. Estes estudos foram desenvolvidos em diversos países, como Estados Unidos (Murphy, 1985, 1999; Abowd, 1990; Leonard, 1990), Reino Unido (Conyon, Gregg \& Machin, 1995; Ozkan, 2011), Itália (Brunello, Graziano \& Parigi, 2001) e Japão (Kato \& Rockel, 1992; Kato \& Kubo, 2006).

De modo geral, constataram que o desempenho e o montante da remuneração dos executivos são correlacionados, porém a sensitividade difere entre países e empresas, pois é influenciada pelas carac- terísticas específicas das nações (Murphy, 1999; Brunello, Graziano \& Parigi, 2001), tamanho da empresa (Kato \& Rockel, 1992; Murphy, 1999; Ozkan, 2011), regulação do setor (Murphy, 1999), estrutura hierárquica (Leonard, 1990), governança corporativa (Ozkan, 2011), origem do controle acionário (Brunello, Graziano \& Parigi, 2001) e nivel de lucratividade (Brunello, Graziano \& Parigi, 2001).

Posteriormente, a literatura empírica centrou sua atenção na identificação do efeito da estrutura de remuneração sobre o desempenho organizacional, uma vez que a relação entre o montante da remuneração e o desempenho, embora existente, era considerada de baixa intensidade (Jensen \& Murphy, 1990b). Neste contexto, destacam-se os estudos de Jensen \& Murphy (1990a) e Mehran (1995), que analisaram o efeito da estrutura de remuneração, tal como o pagamento de remuneração variável $e$ remuneração baseada em ações, sobre o desempenho organizacional.

Empiricamente, os estudos de Jensen \& Murphy (1990a) e Mehran (1995), em empresas norte-americanas, constataram que a forma como a remuneração é composta afeta a motivação dos gestores para a maximização do desempenho das empresas em intensidade maior do que o montante de salário fixo pelo qual os executivos são contratados para a execução das suas atividades administrativas.

Um terceiro estágio da literatura internacional aponta para a análise dos fatores contingenciais que influenciam a estruturação de remuneração, bem como a maneira como estes fatores influenciam a eficiência dos contratos de remuneração para se maximizar o desempenho da organização. Dentre os fatores identificados por esta corrente da literatura estão: estratégia de negócios (Banker et al., 1996; Chen \& Jermias, 2014), controle organizacional (Banker et al., 1996), risco do negócio (Bloom \& Milkovich, 1998), tamanho, descentralização e governança corporativa (Matolcsy; Wright, 2011).

No Brasil, os estudos que relacionaram a remuneração dos executivos e o desempenho das organi- 
zações centram-se, predominantemente, na relação entre o total de remuneração paga e o desempenho da firma. Isto é, verificaram se os gestores que possuem salários mais elevados estão relacionados a um maior desempenho da empresa.

Camargos, Heal \& Boas (2007), Silva \& Chien (2013), Krauter (2013) e Beuren, Silva \& Mazzioni (2014) testaram a hipótese de relacionamento entre o total de remuneração e o desempenho organizacional. Tomados em conjunto, os resultados destas pesquisas apresentam-se como inconclusivos, pois alguns estudos detectaram relação positiva entre o total de remuneração e o desempenho operacional (Camargos, Helal \& Boas, 2007; Krauter, 2013; Castilho, 2015) e de mercado (Beuren, Silva \& Mazzioni, 2014), enquanto Silva \& Chien (2013) concluíram não haver relação tanto sob o aspecto operacional quanto de mercado.

Por outro lado, Nascimento, Franco \& Cherobim (2012) e Nascimento et al. (2013) trouxeram uma nova perspectiva à análise da relação entre sistemas de remuneração e desempenho organizacional na literatura nacional, ao analisarem a relação entre a estrutura de remuneração dos gestores e o desempenho das organizações. Estes estudos compararam se a presença de políticas de remuneração que envolvem elementos variáveis afeta o desempenho das organizações, e ambos constataram não haver relação significativa entre a forma de remuneração e o desempenho.

Nascimento, Franco \& Cherobim (2012) analisaram uma amostra de companhias abertas que atuam no setor de energia elétrica, investigando a presença de remuneração variável. Nascimento et al. (2013) utilizaram como amostra 260 empresas de capital aberto brasileiras, com foco na presença de remuneração baseada em opção de ações. Metodologicamente, ambas as pesquisas centraram suas análises na comparação do desempenho das empresas que possuíam ou não estruturas diferenciadas de remuneração, as quais foram, respectivamente, remuneração variável e remuneração baseada em ações.

Sobre a remuneração variável, destaca-se, ainda, o estudo de Gonzaga, Yoshinaga \& Eid Jr. (2013) que analisaram os determinantes do percentual de remuneração variável das companhias abertas. Os resultados indicam que empresas com maior retorno sobre o patrimônio líquido (ROE) tendem a oferecer menor percentual de remuneração variável na contratação dos executivos, bem como que o desempenho de mercado tende a elevar o grau de remuneração variável praticado pelas empresas.

Diante deste conjunto de resultados, a presente pesquisa posiciona-se como um complemento às evidências empíricas sobre como a remuneração variável relaciona-se com o desempenho organizacional. Especificamente, os resultados desta pesquisa situam-se como um complemento aos achados de Nascimento, Franco \& Cherobim (2012), pois a presente pesquisa avalia se a utilização de percentuais diferenciados de remuneração variável produzem efeitos diferentes sobre o desempenho, enquanto que Nascimento, Franco \& Cherobim (2012) analisaram somente a presença de remuneração variável.

\section{Procedimentos metodológicos}

\subsection{Descrição das variáveis}

\subsubsection{Variáveis dependentes}

Os indicadores de desempenho econômico e financeiro analisados nesta pesquisa dividem-se em dois grupos: a) indicadores utilizados pelos estudos anteriores; e, b) indicadores de desempenho fixados como base para o pagamento da remuneração variável pelas empresas analisadas a seus executivos.

Optou-se pela utilização deste conjunto amplo de indicadores de modo a gerar resultados mais precisos à amostra analisada, pois as organizações podem utilizar métricas diferentes para avaliar o desempenho de seus executivos, o que pode implicar na fraca relação entre os incentivos de remuneração $e$ os indicadores de desempenho geralmente utilizados pela literatura.

Como proxy de desempenho organizacional foi utilizada, inicialmente, a métrica de retorno sobre o ativo médio (ROA). O ROA busca mensurar o desempenho econômico da organização, por meio da 
taxa de lucro contábil gerada no período em relação ao ativo total médio empregado no negócio. Optou-se pela utilização deste indicador, pois ele foi a medida utilizada com maior frequência nos estudos anteriores revisados, o que permite a comparabilidade dos resultados.

Posteriormente, foi realizado um levantamento dos indicadores utilizados como parâmetros para o pagamento de remuneração variável dos executivos e divulgados pelas empresas na sua política de remuneração. Na sequência, os indicadores financeiros apresentados com maior frequência foram utilizados como medida de desempenho para se testar a existência de relação entre o percentual de remuneração variável e estes indicadores.

Após a identificação dos indicadores considerados pelas empresas para o estabelecimento do montante da remuneração variável, foram incluídas mais quatro métricas para a análise do desempenho das empresas: geração de EBITDA; giro do ativo total; geração de fluxo de caixa operacional em relação ao ativo total; e, geração de fluxo de caixa operacional em relação à receita líquida.

Estas métricas foram baseadas nos principais indicadores utilizados pelas empresas para o pagamento de remuneração variável: EBITDA, volume de vendas e geração de fluxo de caixa operacional.

\subsubsection{Variável independente}

A remuneração variável foi analisada pelo seu aspecto de proporção em relação à remuneração total dos gestores. O objetivo desta métrica é identificar se uma maior vinculação da riqueza do gestor à sua performance afeta o desempenho econômico e financeiro da empresa. A partir desta medida, foi realizada a comparação entre o desempenho de gestores com percentuais distintos de remuneração variável.

As informações sobre o percentual de remuneração variável dos executivos foram coletadas da política de remuneração das empresas, divulgada no item 13.1 do Formulário de Referência. Destaca-se que esta análise da remuneração dos executivos se limitou aos profissionais que compõem a diretoria executiva das companhias.

Constatou-se que as empresas não divulgam de forma uniforme as informações sobre a remuneração variável de seus gestores. Algumas empresas evidenciam o percentual estipulado para o período corrente, enquanto outras evidenciam o percentual pago no ano anterior. Diante disto, optou-se pela estimação da política de remuneração da companhia por meio da média dos percentuais divulgados durante os cinco anos analisados, os quais são 2010 a 2014. Este procedimento tem a finalidade de mitigar os efeitos sazonais de períodos mais e menos lucrativos para as companhias que divulgaram os montantes pagos ao invés daqueles previstos.

Ao se analisar a política de remuneração das companhias, foi considerada como remuneração variável aquela que era classificada pela empresa como tal. Diante disto, ela poderia incluir bônus de curto e longo prazo, conforme classificação adotada pela empresa.

\subsubsection{Variáveis de controle}

Nas análises estatísticas, foram incluídas as seguintes variáveis para se controlar as características específicas da firma e que podem ter relação com o nivel de desempenho: Tamanho, Endividamento, Tangibilidade e Setor de atuação. O objetivo da inclusão destas variáveis é controlar os efeitos que a heterogeneidade das empresas pode ter sobre o seu desempenho.

\subsubsection{Operacionalização das variáveis}

A Tabela 1 apresenta as variáveis que foram utilizadas na análise dos dados, bem como o método de mensuração e a fonte de coleta dos dados. 
Tabela 1. Variáveis analisadas na pesquisa

\begin{tabular}{|c|c|c|c|}
\hline Variável & $\begin{array}{l}\text { Tipo da } \\
\text { variável }\end{array}$ & Método de mensuração & Fonte de coleta \\
\hline Retorno sobre 0 ativo médio (ROA) & Dependente & $R O A_{i t}=L O p_{i t} /\left(\left(A T_{i t}+A T_{i t-1}\right) / 2\right)$ & Economatica ${ }^{\circledR}$ \\
\hline $\begin{array}{l}\text { Geração de fluxo de caixa operacional em relação ao } \\
\text { ativo médio }\end{array}$ & Dependente & $\mathrm{FCO}_{\text {it }}=\mathrm{FCO}_{\text {it }} /\left(\left(\mathrm{AT} \mathrm{T}_{\mathrm{it}}+\mathrm{AT_{ \text {itt-1 } } ) / 2 )}\right.\right.$ & Economatica ${ }^{\circledR}$ \\
\hline $\begin{array}{l}\text { Geração de fluxo de caixa operacional em relação à } \\
\text { Receita Líquida }\end{array}$ & Dependente & $\mathrm{FCO}_{\text {it }}=\mathrm{FCO}_{\text {it }} /$ Receita Líquidait & Economatica ${ }^{\circledR}$ \\
\hline Giro do ativo & Dependente & GIR0 $_{\text {it }}=$ Receita líquidait / Ativo totalit & Economatica ${ }^{\circledR}$ \\
\hline Geração de EBITDA & Dependente & $\mathrm{EBITDA}_{\text {it }}=\mathrm{EBITDA}_{\text {it }} /$ Ativo totalit & Economatica ${ }^{\circledR}$ \\
\hline Remuneração & Interesse & Percentual de médio de remuneração variável. & Formulário de Referência \\
\hline Tamanho (InTAM) & Controle & InTAMit $=\operatorname{LnAT}_{\text {it }}$ & Economatica ${ }^{\circledR}$ \\
\hline Tangibilidade (TANG) & Controle & TANG $_{i t}=($ imobilizadoit + estoquesit $) / \mathrm{AT}_{\text {it }}$ & Economatica ${ }^{\circledR}$ \\
\hline Endividamento (END) & Controle & $E N D_{i t}=P E_{i t} / A T_{i t}$ & Economatica ${ }^{\circledR}$ \\
\hline Dummies setoriais & Controle & $\begin{array}{l}\text { Setor de atuação da firma segundo classifi- } \\
\text { cação da Economatica }{ }^{\circledR} \text {. }\end{array}$ & Economatica ${ }^{\circledR}$ \\
\hline $\begin{array}{l}\text { Legenda: LOp: lucro operacional do exercício; AT: ati- } \\
\text { vo total; FCO: fluxo de caixa operacional; PE: passivo } \\
\text { exigível; EBITDA: lucro antes dos juros, impostos, } \\
\text { depreciação e amortização. }\end{array}$ & & & \\
\hline
\end{tabular}

Fonte: Elaborado pelos autores.

\subsection{Amostra e Período de Análise}

A amostra é composta pelas empresas pertencentes ao Índice Brasil 100 (IBrX-100) na data de 23 de novembro de 2015. O IBrX-100 representa uma carteira teórica composta pelas 100 ações mais negociadas da Bolsa de Mercadorias, Valores e Futu- ros de São Paulo (BM\&FBovespa, 2015). Desta carteira, foram excluídas as empresas financeiras, pois as peculiaridades do seu sistema contábil podem causar vieses nos resultados da pesquisa. Com isso, obteve-se uma amostra final de 75 empresas, conforme apresentado na tabela 2 . 
Tabela 2. Amostra da pesquisa

\begin{tabular}{|c|c|c|c|}
\hline 1. AES Tiete & 20. Eletropaulo & 39. Lojas Americanas & 58. Rumo Logística \\
\hline 2. AMBEV & 21. Embraer & 40. Lojas Renner & 59. Sabesp \\
\hline 3. GAEC Educação & 22. Energias do Brasil & 41. M. Dias Branco & 60. São Martinho \\
\hline 4. B2W & 23. Equatorial Energia & 42. Marcopolo & 61. Ser Educacional \\
\hline 5. BRASKEM & 24. Estácio & 43. Marfrig Global Foods & 62. Sid. Nacional \\
\hline 6. BRF & 25. Even & 44. Mills Engenharia & 63. Smiles \\
\hline 7. CCR & 26. Eztec & 45. Minerva & 64. Suzano \\
\hline 8. Cemig & 27. Fibria Celulose & 46. MRV Engenharia & 65. Taesa \\
\hline 9. Cesp & 28. Gafisa & 47. Multiplus & 66. Telefônica Brasil \\
\hline 10. Cia Hering & 29. Gerdau & 48. Natura Cosméticos & 67. Tim \\
\hline 11. Copasa & 30. Metalúrgica Gerdau & 49. Odontoprev & 68. Totvs \\
\hline 12. Copel & 31. Gol & $50.0 \mathrm{i}$ & 69. Tractebel Energia \\
\hline 13. COSAN Indústria e Comércio & 32. Hypermarcas & 51. Pão de açucar & 70. CTEEP \\
\hline 14. Cosan Logística & 33. Iochp-Maxion & 52. Petrobrás & 71. Usiminas \\
\hline 15. CPFL Energia & 34. JBS & 53. Qgep & 72. Vale \\
\hline 16. Cyrela & 35. Klabin & 54. QUALICORP & 73. Valid \\
\hline 17. Duratex & 36. Kroton & 55. Raia Drogasil & 74. Via varejo \\
\hline 18. Ecorodovias & 37. Light & 56. Randon & 75. Weg \\
\hline 19. Eletrobrás & 38. Localiza Rent a Car & 57. Rossi Residencial & \\
\hline
\end{tabular}

Fonte: Elaborado pelos autores.

O período de análise compreendeu os anos de 2010 a 2014, pois no ano de 2010 iniciou a divulgação da política de remuneração dos gestores das companhias de capital aberto brasileiras, por meio do Formulário de Referência.

A coleta dos dados referentes à política de remuneração das empresas foi realizada das informações constantes no Formulário de Referência, item 13.1. Os dados contábeis e de mercado necessários para o cálculo das variáveis dependentes e de controle foram coletados da base de dados do software Economatica ${ }^{\circledR}$. A coleta de dados foi realizada no mês de novembro de 2015 , em que todos os dados financeiros foram corrigidos monetariamente para esta data-base.

\subsection{Modelo estatístico}

Os dados foram analisados por meio de regressões com dados em painel, processadas no software Gretl (Gnu Regression, Econometrics and Time-series Library). O modelo de regressão com dados em painel torna-se mais consistente para este tipo de análise, pois os dados congregam informações observadas ao longo do tempo (séries temporais) de diferentes indivíduos (cross-section) (Fávero, 2013).

O modelo teórico geral testado nesta pesquisa é apresentado na Equação 1. Os resultados desta equação serviram de base para o alcance do objetivo desta pesquisa. Valores positivos (negativos) do coeficiente beta 1 ( $\beta 1$ ) indicam que, em média, o desempenho se apresentou maior (menor) à medida que o percentual de remuneração variável é maior (menor). 


\section{Equação 1:}

Desempenho $_{i t}=\alpha_{i t}+\beta_{1}$ Remuneração $_{i t}+\beta_{2}$ InTamanho $_{i t}+\beta_{3}$ Endividamento $_{\text {it }}$ $+\beta_{4}$ Tangibilidade $_{i t}+\beta_{5}$ Dummies setoriais $_{i t}+u_{i t}$
Foram geradas cinco análises de dados, sendo uma para cada variável de desempenho considerada nesta pesquisa. A diferença entre ambos os modelos é a variável "desempenho", que assumirá o valor do indicador em foco. A tabela 3 apresenta a variável de desempenho considerada especificamente em cada modelo.

Tabela 3. Variáveis de desempenho testadas nos modelos

\begin{tabular}{|l|l|l|}
\hline \multicolumn{1}{|c|}{ Modelo } & \multicolumn{1}{|c|}{ Variável dependente } & \multicolumn{1}{|c|}{ Descrição } \\
\hline Modelo 1 & Retorno sobre 0 ativo médio & Desempenho econômico do período, com foco na rentabilidade. \\
\hline Modelo 2 & Giro do ativo & Desempenho em relação ao volume de vendas. \\
\hline Modelo 3 & $\begin{array}{l}\text { Geração de fluxo de caixa operacional em relação ao } \\
\text { ativo médio }\end{array}$ & $\begin{array}{l}\text { Desempenho financeiro do período, com foco na geração de caixa ope- } \\
\text { racional. }\end{array}$ \\
\hline Modelo 4 & $\begin{array}{l}\text { Geração de fluxo de caixa operacional em relação à Re- } \\
\text { ceita Líquida }\end{array}$ & $\begin{array}{l}\text { Desempenho financeiro do período, com foco na geração de caixa ope- } \\
\text { racional. }\end{array}$ \\
\hline Modelo 5 & Geração de EBITDA em relação ao ativo médio & Desempenho medido pelo potencial de geração de caixa operacional. \\
\hline
\end{tabular}

Fonte: Elaborado pelos autores.

Na sequência, os dados foram submetidos aos testes de Breusch-Pagan, F de Chow e de Hausman para se identificar o estimador em painel mais eficiente.
Adotou-se um nível de significância de $\mathrm{p}<0,01$ para a interpretação dos resultados. A Tabela 4 apresenta os resultados destes testes.

Tabela 4. Resultados dos testes estatísticos para identificação do estimador mais consistente

\begin{tabular}{|c|c|c|c|c|c|}
\hline \multirow{2}{*}{ Teste } & Significância & Significância & Significância & Significância & Significância \\
\hline & Modelo 1 & Modelo 2 & Modelo 3 & Modelo 4 & Modelo 5 \\
\hline Breusch-Pagan & 0,00 & 0,00 & 0,00 & 0,00 & 0,00 \\
\hline F de Chow & 0,00 & 0,00 & 0,07 & 0,48 & 0,00 \\
\hline de Hausman & 0,85 & 0,09 & 0,18 & 0,03 & 0,81 \\
\hline Modelo indicado & Efeitos aleatórios & Efeitos aleatórios & Efeitos aleatórios & Efeitos aleatórios & Efeitos aleatórios \\
\hline
\end{tabular}

Fonte: Elaborado pelos autores.

Com base nos resultados do teste de Breush-Pagan, pode-se considerar que não há adequação do modelo POLS, relativamente ao modelo de efeitos aleatórios para nenhum modelo. Por fim, o teste de Hausman indica que para todos os modelos o estimador de efeitos aleatórios é mais consistente do que o de efeitos fixos.

\section{Análise e interpretação dos resultados}

O objetivo deste estudo é identificar se o percentual de remuneração variável do gestor está relacionado ao aumento do desempenho econômico e financeiro. Ou seja, o objetivo é verificar se o pagamento de maior percentual de remuneração variável está 
positivamente relacionado com o desempenho organizacional.

Para alcançar este objetivo, foram coletadas as informações sobre a remuneração variável dos executivos e dos indicadores de desempenho utilizados pelas companhias como parâmetro para o pagamento desta bonificação. Ambas as informações constam na Política de Remuneração das empresas, divulgada no Formulário de Referência. A Tabela 5 apresenta as estatísticas descritivas da remuneração variável utilizada pelas empresas analisadas.

Tabela 5. Remuneração variável média

\begin{tabular}{|c|c|c|c|c|c|c|}
\hline Média & Desvio padrão & Mínima & Quartil 1 & Mediana & Quartil 3 & Máximo \\
\hline $39,3 \%$ & $17,9 \%$ & $0,0 \%$ & $27,0 \%$ & $40,0 \%$ & $51,0 \%$ & $70,0 \%$ \\
\hline
\end{tabular}

Fonte: Dados da pesquisa.

Verifica-se, na Tabela 5, que, em média, 39\% da remuneração total dos executivos das companhias analisadas está vinculada ao alcance de metas estabelecidas pela política de remuneração. Ademais, destaca-se que apenas duas empresas não utilizam a remuneração variável como componente da estrutura de remuneração dos seus executivos.

Estes dados indicam que a remuneração baseada em compensação é um componente relevante do sistema de incentivos e congruência dos objetivos das em- presas, pois, além de ser amplamente utilizado (97\% das empresas analisadas), ela compreende uma parcela significativa da remuneração dos executivos.

Na sequência, identificou-se os indicadores de desempenho utilizados pelas empresas como parâmetro para a determinação da remuneração variável. Foram identificados 57 indicadores diferentes, os quais foram agrupados de acordo com sua natureza e características de similaridade. A Tabela 6 apresenta os principais indicadores identificados.

Tabela 6. Principais indicadores de desempenho utilizados pelas empresas

\begin{tabular}{|l|c|c|l|c|c|}
\hline \multicolumn{1}{|c|}{ Indicador } & Freq. & Freq. \% & \multicolumn{1}{c|}{ Indicador } & Freq. & Freq. \% \\
\hline EBITDA & 39 & $52 \%$ & Eficiência da produção & 7 & $9 \%$ \\
\hline Lucro líquido & 31 & $41 \%$ & Margem do Ebitda & 7 \\
\hline Volume de vendas & 29 & $39 \%$ & Rentabilidade (ROA, ROE ou ROI) & 7 & $9 \%$ \\
\hline Avaliação desempenho individual & 24 & $32 \%$ & Qualidade dos produtos/serviços & 7 & $9 \%$ \\
\hline Geração de caixa operacional & 17 & $23 \%$ & Acidentes de trabalho & 6 & $8 \%$ \\
\hline Estrutura de custos/despesas & 16 & $21 \%$ & Valor econômico agregado (EVA) & 6 & $8 \%$ \\
\hline Produtividade & 15 & $20 \%$ & Dividendos & 5 & $7 \%$ \\
\hline Satisfação dos clientes & 12 & $16 \%$ & Capital de giro & 4 & $5 \%$ \\
\hline Valor de mercado das ações & 11 & $15 \%$ & Cumprimento do orçamento & 4 & $5 \%$ \\
\hline Cumprimento de metas estratégicas & 11 & $15 \%$ & Segurança operacional & 4 \\
\hline
\end{tabular}

Legenda: Freq. \%: Frequência percentual de empresas que utilizam o indicador (frequência observada / 75 empresas).

Fonte: Dados da pesquisa. 
A partir da Tabela 6, constata-se que os principais indicadores utilizados pelas empresas são os indicadores financeiros EBITDA, Lucro líquido $e$ Volume de Vendas, os quais foram utilizados em mais de um terço das empresas analisadas. Além disto, vários indicadores não financeiros são utilizados para a avaliação do desempenho das metas estabelecidas aos executivos, dentre os quais se destacam o desempenho individual do gestor, a produtividade da organização, a satisfação dos clientes e o cumprimento de metas estratégias fixadas pela organização.

Tomados em conjunto, estes indicadores demonstram que o desempenho fixado como meta pelas empresas é amplo, o que indica que o desempenho dos gestores é avaliado sob vários aspectos de interesse das empresas, os quais englobam benefícios ao seu desempenho econômico e financeiro, bem como a maximização da performance não financeira.

Esta política de remuneração possivelmente é explicada pelo objetivo das empresas em incentivar os gestores a maximizarem o desempenho de diversos aspectos da organização, por conta do efeito conjunto que estes indicadores podem ter sobre o desempenho geral do empreendimento, isto é, na riqueza dos proprietários.

Na sequência, foi realizada a análise da relação entre os indicadores de desempenho financeiros e o percentual de remuneração variável dos executivos da empresa. Para tanto, foram realizadas análises de regressão linear com dados em painel.
Diante da ampla quantidade de indicadores financeiros utilizados pelas empresas como referência para o pagamento da remuneração variável, foi testada nesta pesquisa a relação entre o percentual de remuneração variável com cinco indicadores financeiros de desempenho, os quais são: geração de EBITDA, ROA, giro (volume de vendas) e geração de caixa operacional (escalonado pelo ativo total e escalonado pela receita líquida). Estes foram os indicadores financeiros mais utilizados pelas empresas analisadas, e, por isto, foram adotados como referências de desempenho nas análises estatísticas.

Na Tabela 7 são apresentados os resultados dos testes estatísticos realizados para se analisar a relação entre as variáveis estudadas. A partir dos resultados destes testes poderá ser confirmada ou não a relação apresentada pela literatura revista, de que uma maior vinculação da remuneração do gestor ao seu desempenho (remuneração variável) tende a elevar o desempenho da organização.

Os modelos estimados explicam aproximadamente $40 \%$ da variância dos indicadores de desempenho analisados. Isto indica que ainda existem outros fatores que influenciam o desempenho das organizações, e que não estão presentes nos modelos analisados. Destaca-se, no entanto, que estes fatores podem não ser diretamente observáveis, tais como a conjuntura econômica do período analisado, a forma de concorrência da empresa no mercado em que atua e até as habilidades específicas dos gestores e sua experiência.

Tabela 7. Resultados estatísticos

\begin{tabular}{|c|c|c|c|c|c|c|c|c|c|c|}
\hline \multirow[b]{2}{*}{ Variáveis } & \multicolumn{2}{|c|}{ Modelo 1} & \multicolumn{2}{|c|}{ Modelo 2} & \multicolumn{2}{|c|}{ Modelo 3} & \multicolumn{2}{|c|}{ Modelo 4} & \multicolumn{2}{|c|}{ Modelo 5} \\
\hline & $\begin{array}{l}\text { Coef. } \\
\text { Pad. }\end{array}$ & p-value & $\begin{array}{l}\text { Coef. } \\
\text { Pad. }\end{array}$ & p-value & $\begin{array}{l}\text { Coef. } \\
\text { Pad. }\end{array}$ & p-value & $\begin{array}{l}\text { Coef. } \\
\text { Pad. }\end{array}$ & p-value & $\begin{array}{l}\text { Coef. } \\
\text { Pad. }\end{array}$ & p-value \\
\hline Remuneração variável & $\begin{array}{c}0,06 \\
(0,07)\end{array}$ & 0,40 & $\begin{array}{c}0,39 \\
(0,34)\end{array}$ & 0,25 & $\begin{array}{c}0,01 \\
(0,06)\end{array}$ & 0,95 & $\begin{array}{l}-0,06 \\
(0,12)\end{array}$ & 0,62 & $\begin{array}{c}0,03 \\
(0,05)\end{array}$ & 0,60 \\
\hline Tamanho & $\begin{array}{c}-0,02 \\
(0,01)\end{array}$ & 0,01 & $\begin{array}{l}-0,02 \\
(0,03)\end{array}$ & 0,00 & $\begin{array}{c}-0,02 \\
(0,01)\end{array}$ & 0,02 & $\begin{array}{c}0,00 \\
(0,01)\end{array}$ & 0,97 & $\begin{array}{c}-0,02 \\
(0,01)\end{array}$ & 0,00 \\
\hline Endividamento & $\begin{array}{c}-0,14 \\
(0,04)\end{array}$ & 0,00 & $\begin{array}{c}0,39 \\
(0,15)\end{array}$ & 0,01 & $\begin{array}{c}-0,01 \\
(0,03)\end{array}$ & 0,77 & $\begin{array}{l}-0,15 \\
(0,07)\end{array}$ & 0,02 & $\begin{array}{l}-0,03 \\
(0,03)\end{array}$ & 0,32 \\
\hline
\end{tabular}




\begin{tabular}{|c|c|c|c|c|c|c|c|c|c|c|}
\hline \multirow[b]{2}{*}{ Variáveis } & \multicolumn{2}{|c|}{ Modelo 1} & \multicolumn{2}{|c|}{ Modelo 2} & \multicolumn{2}{|c|}{ Modelo 3} & \multicolumn{2}{|c|}{ Modelo 4} & \multicolumn{2}{|c|}{ Modelo 5} \\
\hline & $\begin{array}{l}\text { Coef. } \\
\text { Pad. }\end{array}$ & p-value & $\begin{array}{l}\text { Coef. } \\
\text { Pad. }\end{array}$ & p-value & $\begin{array}{l}\text { Coef. } \\
\text { Pad. }\end{array}$ & p-value & $\begin{array}{l}\text { Coef. } \\
\text { Pad. }\end{array}$ & p-value & $\begin{array}{l}\text { Coef. } \\
\text { Pad. }\end{array}$ & p-value \\
\hline Tangibilidade & $\begin{array}{c}-0,02 \\
(0,03)\end{array}$ & 0,54 & $\begin{array}{l}-0,01 \\
(0,08)\end{array}$ & 0,97 & $\begin{array}{c}0,04 \\
(0,02)\end{array}$ & 0,04 & $\begin{array}{c}0,14 \\
(0,04)\end{array}$ & 0,00 & $\begin{array}{c}0,05 \\
(0,02)\end{array}$ & 0,02 \\
\hline Constante & $\begin{array}{c}0,65 \\
(0,19)\end{array}$ & 0,00 & $\begin{array}{l}3,06 \\
(0,8)\end{array}$ & 0,00 & $\begin{array}{c}0,51 \\
(0,17)\end{array}$ & 0,00 & $\begin{array}{c}0,19 \\
(0,32)\end{array}$ & 0,55 & $\begin{array}{c}0,52 \\
(0,14)\end{array}$ & 0,00 \\
\hline Dummies setores & \multicolumn{2}{|c|}{ Incluídas } & \multicolumn{2}{|c|}{ Incluídas } & \multicolumn{2}{|c|}{ Incluídas } & \multicolumn{2}{|c|}{ Incluídas } & \multicolumn{2}{|c|}{ Incluídas } \\
\hline $\mathrm{R}^{2}$ ajustado & \multicolumn{2}{|c|}{$31 \%$} & \multicolumn{2}{|c|}{$56 \%$} & \multicolumn{2}{|c|}{$35 \%$} & \multicolumn{2}{|c|}{$40 \%$} & \multicolumn{2}{|c|}{$36 \%$} \\
\hline Wald chi2 / Teste F & \multicolumn{2}{|c|}{$46,61^{* * *}$} & \multicolumn{2}{|c|}{$105,3 * \star \star$} & \multicolumn{2}{|c|}{$35,4^{* *}$} & \multicolumn{2}{|c|}{$55,2 * \star \star$} & \multicolumn{2}{|c|}{$51,4^{* * *}$} \\
\hline p-value Jarque-Bera & \multicolumn{2}{|c|}{0,01} & \multicolumn{2}{|c|}{0,00} & \multicolumn{2}{|c|}{0,00} & \multicolumn{2}{|c|}{0,00} & \multicolumn{2}{|c|}{0,00} \\
\hline Observações (N) & \multicolumn{2}{|c|}{352} & \multicolumn{2}{|c|}{352} & \multicolumn{2}{|c|}{351} & \multicolumn{2}{|c|}{351} & \multicolumn{2}{|c|}{350} \\
\hline Empresas (n) & \multicolumn{2}{|c|}{75} & \multicolumn{2}{|c|}{75} & \multicolumn{2}{|c|}{75} & \multicolumn{2}{|c|}{75} & \multicolumn{2}{|c|}{75} \\
\hline
\end{tabular}

Legenda: ${ }^{* * * *},{ }^{* *}, *$; Significante a $1 \%, 5 \%$ e 10\%, respectivamente. O valor entre parênteses representa o erro-padrão do coeficiente beta estimado. Modelo 1: ROA; Modelo 2: Giro; Modelo 3: geração de caixa operacional sobre ativo médio; Modelo 4: geração de caixa operacional sobre receita líquida; Modelo 5: Geração de EBITDA em relação ao ativo médio.

Fonte: Dados da pesquisa.

A partir análise da Tabela 7 pode-se observar que, para as empresas analisadas nesta amostra, o percentual de remuneração variável não apresentou relação estatisticamente significante com os indicadores de desempenho, assumindo-se um nivel de confiança de $90 \%$.

Tomados em conjunto, estes resultados indicam que, para a amostra analisada, o percentual de remuneração variável não apresentou uma relação direta com o desempenho econômico e financeiro das empresas. Diante disto, pode-se interpretar que a atribuição de um maior percentual de remuneração na forma variável aos gestores não gera um impacto direto sobre o desempenho econômico $e$ financeiro dessas empresas.

Estes resultados não confirmam empiricamente as proposições teóricas contidas na literatura, as quais sugerem que a utilização da remuneração variável tende a incentivar os gestores a investirem em projetos que maximizem a performance da organização e, por conseguinte, tende a elevar o desempenho organizacional (Smith \& Watts, 1982; Eisenhardt, 1989; Hirshleifer \& Suh, 1992). Ademais, estes resultados também não corroboram os resultados empíricos encontrados por Jensen \&
Murphy (1990a) e Mehran (1995) na análise das companhias norte-americanas.

Embora estes resultados não confirmem a relação teórica descrita na literatura, eles estão alinhados aos estudos anteriores realizados no Brasil que analisaram a relação entre a remuneração variável e o desempenho organizacional. Especificamente, Nascimento, Franco \& Cherobin (2012) também não constataram relação estatisticamente significante entre a presença de remuneração variável e o desempenho organizacional.

Assim, pode-se concluir, a partir destes novos resultados, de que tanto a presença de remuneração variável quanto o percentual oferecido como remuneração variável não provocam um efeito diretamente observável sobre o desempenho econômico e financeiro das organizações.

Destaca-se, no entanto, que os dados coletados sobre os indicadores de desempenho utilizados pelas empresas para a determinação do valor pago na forma de remuneração variável a seus executivos fornecem alguns indícios sobre os possiveis fatores explicativos para esta ausência de relação empírica. 
Uma possivel explicação para isto é de que a remuneração variável dos gestores é baseada em diversas medidas de desempenho, as quais podem diferir entre empresas. Assim, é possivel que a utilização de maiores percentuais de remuneração variável exerça um papel de incentivo ao gestor elevar o desempenho em diversos pontos específicos da empresa, porém, esta amplitude de indicadores não permite que ela seja observada estatisticamente por meio de um único indicador de desempenho, o que resulta em uma fraca relação nos modelos analisados.

Além disto, a ampla quantidade de indicadores pode gerar um incentivo aos executivos a maximizarem o desempenho de outros aspectos da companhia que não foram aqueles necessariamente analisados por esta pesquisa, ou pelas pesquisas anteriores.

Portanto, como nem todas as empresas utilizam os mesmos indicadores de desempenho, é possivel que os esforços dos gestores não se reflitam uniformemente nos mesmos indicadores de desempenho analisados, o que também pode explicar a fraca relação entre o percentual de remuneração variável $e$ os indicadores específicos de desempenho.

Por fim, deve-se considerar ainda como um possivel fator explicativo para a ausência de relação o fato de a parcela fixa de remuneração total recebida pelos gestores ser de grande montante. Assim, o conforto gerado pelo recebimento da alta remuneração fixa pode tornar a remuneração variável menos atrativa para o gestor buscar resultados superiores.

\section{Conclusões}

Este estudo teve como objetivo identificar se o percentual da remuneração dos executivos baseada em forma variável possui relação com os indicadores de desempenho econômico e financeiros. Os resultados encontrados indicam que não há uma relação estatisticamente significante entre o percentual de remuneração variável e o desempenho econômico e financeiro das empresas.

Especificamente, os resultados indicam que a atribuição de maiores percentuais de remuneração variável aos gestores não gera necessariamente um aumento do desempenho da organização. Uma das possiveis explicações para a fraca relação encontrada nos testes estatísticos é a grande diversidade de indicadores considerados para a determinação do montante a ser pago como remuneração variável. É possivel que a remuneração variável exerça o papel de incentivo aos gestores maximizarem o desempenho das organizações em que atuam, porém estes concentram seus esforços em outros indicadores, tal como os não financeiros.

Diante disto, sugere-se que a pesquisa sobre remuneração variável e desempenho siga um novo panorama para obter resultados mais precisos, tal como a realização de testes via procedimentos de experimentação. Isto se dá, pois, nas empresas com dados disponíveis ao público externo, a remuneração variável é vinculada a diversos indicadores de desempenho organizacional, os quais apresentam indícios de baixa adequação aos métodos estatísticos utilizados nesta pesquisa.

As contribuições que este estudo traz à literatura de remuneração variável e desempenho é que a relação pode não ser diretamente observada, pois são diversos os aspectos levados em consideração para se medir o desempenho vinculado à remuneração variável.

Além disto, esta pesquisa gera uma relevante contribuição no âmbito acadêmico, nas disciplinas de gestão de pessoas, uma vez que apresenta que, na prática empresarial, as organizações adotam diversas áreas de resultados para se mensurar o desempenho dos gestores e, assim, atribuir a remuneração variável.

Este fenômeno pode ser explicado devido à necessidade de se garantir que os gestores se esforcem para a maximização do resultado da organização de uma forma holística ao invés de se concentrarem em um único aspecto. A concentração da remuneração variável em um único indicador de desempenho poderia causar desequilíbrio nas demais áreas de resultados, o que geraria gargalos na performance e tenderia a implicar na redução do desempenho geral da organização.

Destaca-se, ainda, que este estudo possui algumas limitações metodológicas, as quais devem ser ana- 
lisadas com cautela no momento da generalização dos resultados. A primeira delas é que se analisou como amostra as empresas do $\mathrm{IBrX}-100$, as quais tendem a ser empresas com elevada representatividade de valor de mercado e foram selecionadas de forma não aleatória. Assim, os resultados desta pesquisa podem diferir daquelas empresas que não pertencem a este índice. A segunda refere-se aos indicadores de desempenho analisados, os quais não englobam todos os aspectos de desempenho econômico e financeiro disponiveis na literatura.

Assim, sugere-se para futuras pesquisa a realização de análises das empresas que não são componentes do IBrX-100 para verificar se o comportamento da relação é o mesmo nas empresas com menor volume de negociação de ações. Outra possibilidade de estudo é analisar o comportamento das variáveis dentro dos setores específicos para verificar se os resultados são uniformes entre os setores. Por fim, sugere-se a análise deste problema de pesquisa por meio de outros designs de pesquisa, tal como entrevistas ou de pesquisa do tipo experimento.

\section{Referências}

Abowd, J. M. (1990). Does performance-based managerial compensation affect corporate performance?. En: Industrial \& Labor Relations Review, 43(3): 52-S-73-S.

Aguiar, A. B. de, Teixeira, A. J. C., Nossa, V., \& Gonzaga, R. P. (2012). Associação entre sistema de incentivos gerenciais e práticas de contabilidade gerencial. En: Revista de Administração de Empresas, 52(1): 40-54.

Banker, R. D., Lee, S.-Y., Potter, G., \& Srinivasan, D. (1996). Contextual Analysis of Performance Impacts of OutcomeBased Incentive Compensation. En: Academy of Management Journal, 39(4): 920-948.

Beuren, I. M., Silva, M. Z. da, \& Mazzioni, S. (2014). Remuneração dos executivos versus desempenho das empresas. En: Revista de Administração FACES Journal, 13(2): 8-25.

Bloom, M., \& Milkovich, G. T. (1998). Relationships among risk, incentive pay, and organizational performance. En: Academy of Management Journal, 41(3): 283-297.

BM\&FBovespa - Bolsa de Valores, Mercadorias e Futuros de São Paulo (Recuperado em 23 de novembro, 2015). Índice Brasil 100 - IBrX 100. Disponivel em: http://www. bmfbovespa.com.br/indices/ResumoCarteiraTeorica. aspx?Indice $=$ IBRX\&idioma $=$ pt-br.

Bonner, S. E., \& Sprinkle, G. B. (2002). The effects of monetary incentives on effort and task performance: Theories, evidence, and a framework for research. En: Accounting, Organizations and Society, 27(4-5): 303-345.
Brunello, G., Graziano, C., \& Parigi, B. (2001). Executive compensation and firm performance in Italy. En: International Journal of Industrial Organization, 19(1-2): 133-161.

Camargos, M. A. De, Helal, D. H., \& Boas, A. P. (2007). Análise empírica da relação entre a remuneração de executivos e o desempenho financeiro de empresas brasileiras. En: XXVII Encontro nacional de engenharia de produção. Foz do Iguaçu - PR, Brasil.

Castilho, Z. H. R. de. (2015). Relação entre a Remuneração de Executivos e o Desempenho Financeiro dos Bancos Brasileiros de Capital Aberto. En: XV Congresso USP de Controladoria e Contabilidade. São Paulo - SP, Brasil.

Chen, Y., \& Jermias, J. (2014). Business strategy, executive compensation and firm performance. En: Accounting \& Finance, 54(1): 113-134.

Conyon, M., Gregg, P., \& Machin, S. (1995). Taking Care of Business: Executive Compensation in the United Kingdom. En: The Economic Journal, 105(430): 704.

Eisenhardt, M. (1989). Agency Theory : and Assessment Review. En: The Academy of Management, 14(1): 57-74.

Fávero, L. P. L. (2013). Dados em painel em contabilidade e finanças: teoria e aplicação. En: Brazilian Business Review, 10(1): 131-156.

Gonzaga, R. P., Yoshinaga, C. E., \& Eid Junior, W. (2013). Sistemas de Incentivos Oferecidos aos Gestores e Desempenho das Empresas Brasileiras. En: Revista Contabilidade Vista \& Revista, 24(3): 103-118.

Hirshleifer, D., \& Suh, Y. (1992). Risk, managerial effort, and project choice. En: Journal of Financial Intermediation, 2(3): 308-345.

Ittner, C. D., Larcker, D. F., \& Rajan, M. V. (1997). The choice of performance measures in annual bonus contracts. En: Accounting Review, 72(2): 231-255.

Jensen, M. C., \& Meckling, W. H. (1976). Theory of the firm: Managerial behavior, agency costs and ownership structure. En: Journal of Financial Economics, 3(4): 305-360.

Jensen, M. C., \& Murphy, K. J. (1990a). CEO incentives - It's not how much you pay, but how. En: Journal of Applied Corporate Finance, 3(3): 36-49.

Jensen, M. C., \& Murphy, K. J. (1990b). Performance Pay and Top-Management Incentives. En: Journal of Political Economy, 98(2): 225-264.

Kato, T., \& Kubo, K. (2006). CEO compensation and firm performance in Japan: Evidence from new panel data on individual CEO pay. En: Journal of the Japanese and International Economies, 20(1): 1-19.

Kato, T., \& Rockel, M. (1992). Experiences, credentials, and compensation in the Japanese and U.S. managerial labor markets: Evidence from new micro data. En: Journal of the Japanese and International Economies, 6(1): 30-51.

Krauter, E. (2013). Remuneração de Executivos e Desempenho Financeiro: um Estudo com Empresas Brasileiras. En: Revista de Educação E Pesquisa Em Contabilidade, 7(3): 259-273.

Leonard, J. (1990). Executive pay and firm performance. En: Industrial \& Labor Relations Review, 43(3): 13-29. 
Matolcsy, Z., \& Wright, A. (2011). CEO compensation structure and firm performance. En: Accounting and Finance, 51(3): 745-763.

Mehran, H. (1995). Executive compensation structure, ownership, and firm performance. En: Journal of Financial Economics, 38(2): 163-184.

Merchant, K. a., Van der Stede, W. a., \& Zheng, L. (2003). Disciplinary constraints on the advancement of knowledge: The case of organizational incentive systems. En: Accounting, Organizations and Society, 28(2-3): 251-286.

Murphy, K. J. (1985). Corporate performance and managerial remuneration. En: Journal of Accounting and Economics, 7(1-3): 11-42.

Murphy, K. J. (1999). Executive compensation. En: Handbook of Labor Economics, 3 Part(2): 2485-2563.

Nascimento, C. Do, Franco, L. M. G., \& Cherobim, A. P. M. S. (2012). Associação Entre Remuneração Variável e Indicadores Financeiros: Evidências do Setor Elétrico. En: Revista Universo Contábil, 8(1): 22-36.
Nascimento, E. M., Barbosa Neto, J. E., da Cunha, J. V. A., \& Dias, W. de O. (2013). Teoria da agência e remuneração de executivos: influência do uso de stock options no desempenho das empresas brasileiras. En: Revista Iberoamericana de Contabilidad de Gestión, XI(21): 1-16.

Ozkan, N. (2011). CEO Compensation and Firm Performance: An Empirical Investigation of UK Panel Data. En: European Financial Management, 17(2): 260-285.

Reichelstein, S. (2000). Providing Managerial Incentives: Cash Flows versus Accrual Accounting. En: Journal of Accounting Research, 38(2): 243-269.

Silva, A. L. C. Da, \& Chien, A. C. Y. (2013). Remuneração Executiva, Valor e Desempenho das Empresas Brasileiras Listadas. En: Revista Brasileira de Finanças, 11(4): 481-502.

Smith, C. W., \& Watts, R. L. (1982). Incentive and Tax Effects of Executive Compensation Plans. En: Australian Journal of Management, 7(2): 139-157.

Smith, C., \& Stulz, R. (1985). The determinants of firm's hedging policies. En: Journal of Financial and Quantitive Analysis: 20(4). 